\title{
Fuzzy Descriptors based on Color, Coarseness, Directionality and Contrast for Image Retrieval
}

\author{
J.M. Soto-Hidalgo ${ }^{1}$ P.M. Martínez-Jiménez ${ }^{2}$ J. Chamorro-Martínez $^{2}$ \\ ${ }^{1}$ Department of Computer Architecture, Electronics and Electronic Technology, Univ. of Córdoba, Spain \\ ${ }^{2}$ Department of Computer Science and Artificial Intelligence, University of Granada, Spain
}

\begin{abstract}
In this paper the concept of fuzzy descriptor as a level-two fuzzy set to represent some visual features is proposed. In particular, a fuzzy descriptor based on the dominance of color and texture features is defined and applied to image retrieval. For this purpose, the color and texture are modelled using fuzzy sets, taking into account the imprecision related to these visual features. In addition, the dominance is defined on the basis of a nondecreasing fuzzy quantifier, and the degree of dominance is calculated by means of quantified sentences evaluation. Finally, comparison measures between fuzzy descriptors are presented, and the proposed descriptors and measures are illustrated in image retrieval examples.
\end{abstract}

Keywords: Fuzzy Descriptor, Color modelling, Texture modelling, Quantified sentences, Retrieval

\section{Introduction}

In last years, large collections of digital images have been created. Usually, the only way of searching these collections was by keyword indexing based on captions and textual descriptors performed by humans [1]. Although this is a useful way to describe images, its main drawback is the requirement of a person who makes the description (subjective, in any case). This fact has motivated an increment of the research about techniques for storing, indexing and retrieving visual information.

The current image retrieval systems improve the textual-based ones by means of features, such as color, texture or shape, which are automatically extracted from images [2]. In these systems, images are represented by vectors of features, queries are defined as an image or sketch, and the matching between them is performed by measuring the similarity of the corresponding vectors.

In this framework, a very important point to take into account is the imprecision in the feature descriptions, as well as the store and retrieval of that imprecise data. To deal with this vagueness, some interesting approaches introduce the use of fuzzy logic in the feature representation and in the retrieval process $[3,4]$. In these fuzzy approaches the semantic data is managed by means of fuzzy sets, allowing to perform queries on the basis of linguistic terms. However, these approaches have two main drawbacks: (i) given a feature, the fuzzy sets are not obtained by considering the relationship between the computational feature and its human perception $[5,6]$ so the linguistic labels related to these fuzzy sets do not necessarily match what a human would expect; and (ii), from our knowledge, none of these approaches propose fuzzy descriptors of features for describing semantically an image [7] so all imprecise information that fuzzy sets provides is not considered in the retrieval process.

In this paper, we face the previous questions introducing fuzzy descriptors for colors and textures. For color representation, the fuzzy color and fuzzy color space definitions we introduced in [8] will be used. Concretely, a color will be modelled by means of a fuzzy set (a fuzzy color) and a fuzzy partition will be defined in the color feature domain (a fuzzy color space). In this paper we propose to define this partition on the basis of the ISCC-NBS color naming system [9], that is based on the human perception of color.

For texture representation, we will use the properties of coarseness, contrast and directionality, that, according to the psychological experiments performed by Tamura et al. in [10], are considered the three most important texture properties for human perception $[11,12]$. In fact, visual textures are usually described by humans using linguistic terms like "very coarse", "low directional", or "high contrasted". In this paper, we propose to model these perceptual properties by means of fuzzy sets. Concretely, fuzzy partitions on the domain of some of the most representative measures of each property are proposed, where the number of linguistic labels and the parameters of the membership functions will be calculated by relating the values given by the measures (our reference set) with the human perception of the corresponding property.

Fuzzy descriptors based on the dominance of color and texture features are defined in order to describe images semantically. The dominance of each fuzzy property (color and texture) is calculated on the basis of (i) a fuzzy quantifier representing the notion of dominance, and (ii) a fuzzy histogram based on gradual numbers, which represents the percentage of pixels that match each fuzzy property as a fuzzy quantity. Finally, comparison measures between fuzzy descriptors is also proposed and illustrated in image retrieval examples. 
The rest of the paper is organized as follows. In sections 2.1 and 2.2 the color and texture fuzzy modellings, presented in our previos works [8, 13], are summarized. In section 3 a fuzzy descriptor and comparison measures between them are proposed. The dominance-based color and texture fuzzy descriptors are defined in section 4. Results of applying dominance-based fuzzy descriptor and comparison measures are shown in section 5, and the main conclusions and future work are summarized in section 6 .

\section{Fuzzy Modelling of Visual Features}

Images can be described by several visual features. However, these features are often imprecise. In this section, we propose two approaches to model color and texture features using fuzzy sets.

\subsection{Fuzzy Modelling of Colors}

In this section, the notions of fuzzy color (section 2.1.1) and fuzzy color space (section 2.1.2) we presented in a previous work [8] are summarized. Based on it, a fuzzy partition will be defined in the color feature domain (our fuzzy color space) according to the ISCC-NBS color naming system [9].

\subsubsection{Fuzzy color}

The color can be represented computationally on the basis of vector spaces, also known as color spaces. In this sense, colors are represented as a triplet of real numbers in a coordinate system. However, as we have mentioned in previous works $[8,6]$, the color is imprecise. In order to manage the imprecision in color description, we introduce the following definition of fuzzy color:

Definition 2.1 A fuzzy color $\widetilde{C}$ is a linguistic label whose semantics is represented as a normal fuzzy subset of colors.

Imposing the use of normal membership functions implies that for each fuzzy color $\widetilde{C}$ there is at least one crisp color $\mathbf{r}$ such that $\widetilde{C}(\mathbf{r})=1$. As a consequence, no fuzzy color is represented by the empty set. We require normalization since we expect that at least one color is fully representative of a color category.

Notice that in definition 2.1 there is no restriction about how crisp colors in the support of a fuzzy color are represented in the computer, though they all will usually be represented using one single designated crisp color space for convenience.

In this paper, and following [8], we will define the membership function of $\widetilde{C}$ as

$$
\widetilde{C}(\mathbf{c} ; \mathbf{r}, S, \Omega)=f\left(|\overrightarrow{\mathbf{r c}}| ; t_{1}^{c}, \ldots, t_{n}^{c}\right)
$$

depending on there parameters: $S=\left\{S_{1}, \ldots, S_{n}\right\}$ a set of bounded surfaces in a color space verifying $S_{i} \cap S_{j}=\emptyset \forall i, j$ (i.e., pairwise disjoint)

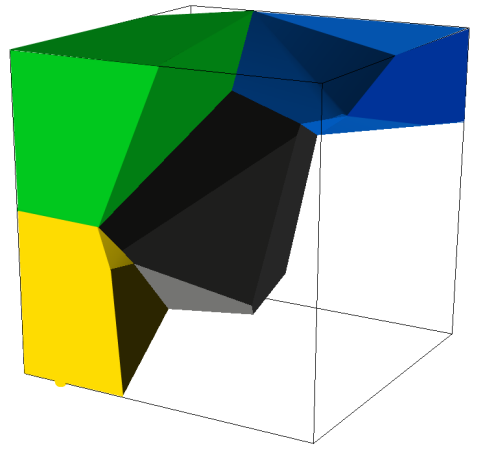

(a)

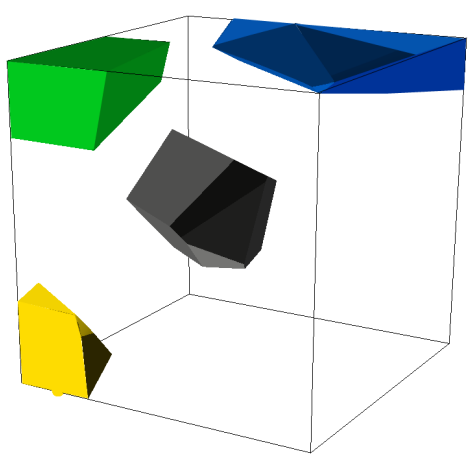

(b)

Figure 1: Some surfaces associated to the fuzzy color space. (a) $S_{2}^{i}$ (b) $S_{1}^{i}$ for $i=$ $\{$ yellow, blue, green, grey\}

and such that $\operatorname{Volume}\left(S_{i}\right) \subset \operatorname{Volume}\left(S_{i+1}\right) ; \Omega=$ $\left\{\alpha_{1}, \ldots, \alpha_{n}\right\} \subseteq(0,1]$, with $1=\alpha_{1}>\alpha_{2}>\cdots>$ $\alpha_{n}=0$, the membership degrees associated to $S$ verifying $\widetilde{C}(\mathbf{s} ; \mathbf{r}, S, \Omega)=\alpha_{i} \forall \mathbf{s} \in S_{i}$; and $\mathbf{r}$ a point inside $\operatorname{Volume}\left(S_{1}\right)$ that is assumed to be a crisp color representative of $\widetilde{C}[8]$.

In Eq.1, $f: \mathbb{R} \rightarrow[0,1]$ is a piecewise function with knots $\left\{t_{1}^{c}, \ldots, t_{n}^{c}\right\}$ verifying $f\left(t_{i}^{c}\right)=\alpha_{i} \in \Omega$, where these knots are calculated from the parameters $\mathbf{r}$, $S$ and $\Omega$ as follows: $t_{i}^{c}=\left|\overrightarrow{\mathbf{r}} \overrightarrow{\mathbf{p}}_{i}\right|$ with $\mathbf{p}_{i}=S_{i} \cap \overline{\mathbf{r c}}$ being the intersection between the line $\overline{\mathbf{r c}}$ (straight line containing the points $\mathbf{r}$ and $\mathbf{c}$ ) and the surface $S_{i}$, and $\left|\overrightarrow{\mathbf{r}} \mathbf{p}_{i}\right|$ the length of the vector $\overrightarrow{\mathbf{r}} \overrightarrow{\mathbf{p}}_{i}[8]$.

\subsubsection{Fuzzy color space}

For extending the concept of color space to the case of fuzzy colors, and assuming a fixed color space $\Gamma$, the following definition is introduced:

Definition 2.2 A fuzzy color space $\widetilde{\Gamma}$ is a set of fuzzy colors.

As we introduced in the previous section (see Eq.1), each fuzzy color $\widetilde{C}_{i} \in \widetilde{\Gamma}$ will have associated a representative crisp color $\mathbf{r}_{i}$. Therefore, for defining our fuzzy color space, a set of representative crisp colors $R=\left\{\mathbf{r}_{1}, \ldots, \mathbf{r}_{n}\right\}$ is needed. In this paper we propose to use the color names (and 
color points) provided by the ISCC-NBS system [9], witch is based on human tests about color perception. ISCC-NBS system defines a set of valid terms and modifiers which can be combined to obtain the final color name. The basic set is formed by 13 color names (10 hues -pink, red, orange, brown, olive, green, blue, violet, purple- and 3 achromatic colors -white, grey and black-), while the extended set is formed by 31 colors (the basic ones and some combination of them formed by adding the $-i s h$ suffix -Brownish Orange, Purplish Blue among others-). In this paper, the extended set will be used (i.e, $R=\left\{\mathbf{r}_{1}, \ldots, \mathbf{r}_{31}\right\}$ with $\mathbf{r}_{i}$ a RGB color). For example, Figure 1 shows the surfaces $S_{2}^{\text {yellow }}, S_{2}^{\text {blue }}$, $S_{2}^{\text {green }}$ and $S_{2}^{\text {grey }}$ (Figure 1(a)), and $S_{1}^{\text {yellow }}, S_{1}^{\text {blue }}$, $S_{1}^{\text {green }}$ and $S_{1}^{\text {grey }}$ (Figure 1(b)) [8].

\subsection{Fuzzy Modelling of Texture Properties}

In this section, the fuzzy modelling of textures is presented. For this modelling, the methodology proposed in our previous work [13] for the coarseness property has been extended to contrast and directionality. Given a texture property (coarseness, contrast, directionality, regularity, etc), let $\mathcal{M}$ be a measure of that property and let $\mathcal{D}_{\mathcal{M}}$ be the measure domain ${ }^{1}$. As in the case of the color modelling shown in section 2.1, the notions of fuzzy texture and fuzzy texture space are introduced as follows:

Definition 2.3 A fuzzy texture $\widetilde{T}$ is a linguistic label whose semantics is represented by a normalized fuzzy subset of $\mathcal{D}_{\mathcal{M}}$.

Definition 2.4 A fuzzy texture space $\Pi$ is a set of fuzzy textures that defines a partition of $\mathcal{D}_{\mathcal{M}}$.

By using these concepts, the fuzzy modelling of coarseness, contrast and directionality properties is described below. From now on, let $\mathcal{P}=\{$ coarseness, contrast, directionality $\}$ be the set of texture properties that will be modelled in this paper. As it was pointed, we propose to model these properties by means of fuzzy partitions defined on the domain of representative computational measures (our fuzzy texture space). Thus, given a measure $\mathcal{M}_{k}^{p}$ of a property $p \in \mathcal{P}$, we propose to define a fuzzy partition $\Pi_{k}^{p}$ on the domain of the measure, obtaining linguistic labels associated to the property (our fuzzy textures). From now on, let $N_{k}^{p}$ be the number of fuzzy sets which compounds the partition $\Pi_{k}^{p}$, and let $T_{k, i}^{p}$ be the i-th fuzzy set in $\Pi_{k}^{p}$. In our approach, we propose to define the membership function $\widetilde{T}_{k, i}^{p}(x)$ for each fuzzy set $\widetilde{T}_{k, i}^{p}$ by using a trapezoidal function of the form

\footnotetext{
${ }^{1}$ Usually, in most popular measures, $\mathcal{D}_{\mathcal{M}}=\mathbb{R}$
}

$$
\widetilde{T}_{k, i}^{p}(x)= \begin{cases}0 & x<a_{k}^{i} \quad \text { or } \quad x>d_{k}^{i} \\ \frac{x-a_{k, i}^{p}}{b_{k, i}^{p}-a_{k, i}^{p}} & a_{k}^{i} \leq x \leq b_{k}^{i} \\ 1 & b_{k}^{i} \leq x \leq c_{k}^{i} \\ \frac{d_{k, i}^{p}-x}{d_{k, i}^{p}-c_{k, i}^{p}} & c_{k}^{i} \leq x \leq d_{k}^{i}\end{cases}
$$

It should be noticed that $a_{k, 1}^{p}=b_{k, 1}^{p}=-\infty$ and $c_{k, N_{k}}^{p}=d_{k, N_{k}}^{p}=\infty$.

This way, two questions need to be faced in order to define the fuzzy partition $\Pi_{k}^{p}$ : (i) how many fuzzy sets will compound the partition, and (ii) how to obtain the parameter values of the membership function for each fuzzy set. In order to solve these questions, we propose a solution based on the study shown in [13].

With regard to the number of fuzzy sets which compounds the partition, we propose to analyze the ability of each measure to distinguish between different presence degrees of the corresponding property. This analysis is based on how the human perceives visual texture. To get information about the human perception of a property $p \in \mathcal{P}$, a set of images covering different degrees of this property is gathered. These images are used to collect, by means of a poll, human assessments about the presence of $p$. Using the data about human perception, and the measure values obtained for each image, an iterative algorithm based on a set of multiple comparison tests is applied in order to obtain the number of classes (presence degrees of the property) that each measure can discriminate. Thus, we propose to set the number of fuzzy sets $N_{k}^{p}$ in the partition $\Pi_{k}^{p}$ as the number of classes that can be discriminated by the measure $\mathcal{M}_{k}^{p}$. In the case of the coarseness property, the detailed description of the analyzed measures, the set of images, the poll and the iterative algorithm is shown in our previous work [13]. In this paper, similar studies have been performed for the properties of contrast and directionality.

In addition, for each fuzzy set $\widetilde{T}_{k, i}^{p}$, we propose to define the parameters of the corresponding membership function $\widetilde{T}_{k, i}^{p}(x)$ on the basis of the information given by the tests. For each class obtained in the distinguishability analysis, its representative value is computed as the mean of the measure values in the class. In our proposal, the center of the kernel of the fuzzy set $\widetilde{T}_{k, i}^{p}$ is established by the representative value of the corresponding i-th class given by the tests. The kernel size will be set as the size of the confidence interval around this representative value. Thus, since a fuzzy partition in the sense of Ruspini is proposed, all the parameter values are obtained. 


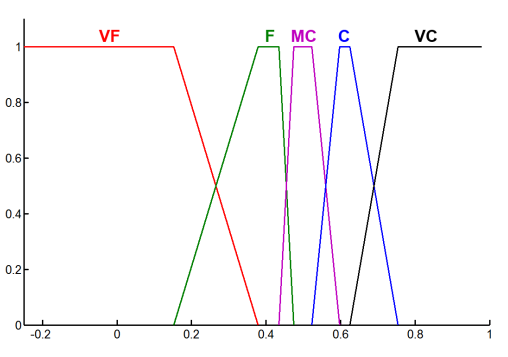

\begin{tabular}{ccccc}
\multicolumn{6}{c}{ Fineness (Correlation) } \\
\hline $\mathrm{i}$ & $a_{k}^{2}$ & $b_{k}^{2}$ & $c_{k}^{2}$ & $d_{k}^{2}$ \\
\hline 1 & $-\infty$ & $-\infty$ & 0.1600 & 0.3758 \\
2 & 0.1600 & 0.3758 & 0.4302 & 0.4725 \\
3 & 0.4302 & 0.4725 & 0.5175 & 0.5937 \\
4 & 0.5175 & 0.5937 & 0.6203 & 0.7480 \\
5 & 0.6203 & 0.7480 & $\infty$ & $\infty$ \\
\hline
\end{tabular}

Linguistic labels:

VC: "very coarse", C: "coarse", MC: "medium coarse", F: "fine", VF: "very fine"

(a)
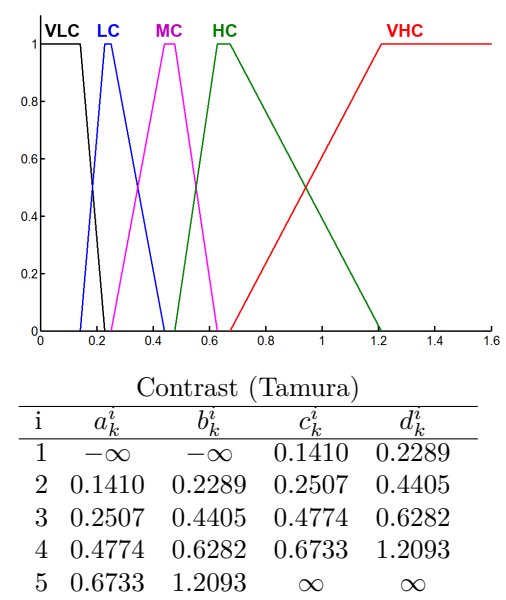

Linguistic labels:

VLC: "very low contrasted", LC: "low contrasted", MC: "medium contrasted", HC: "high contrasted", VHC: "very high contrasted"

(b)

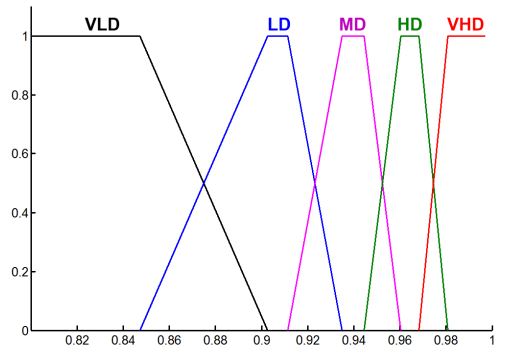

\begin{tabular}{ccccc}
\multicolumn{5}{c}{ Directionality (Tamura) } \\
\hline $\mathrm{i}$ & $a_{k}^{\imath}$ & $b_{k}^{2}$ & $c_{k}^{2}$ & $d_{k}^{2}$ \\
\hline 1 & $-\infty$ & $-\infty$ & 0.8471 & 0.9027 \\
2 & 0.8471 & 0.9027 & 0.9113 & 0.9350 \\
3 & 0.9113 & 0.9350 & 0.9445 & 0.9604 \\
4 & 0.9445 & 0.9604 & 0.9682 & 0.9807 \\
5 & 0.9682 & 0.9807 & $\infty$ & $\infty$ \\
\hline
\end{tabular}

Linguistic labels:

VLD: "very low directional", LD: "low directional", MD: "medium directional", HD: "high directional", VHD: "very high directional"

(c)

Figure 2: Proposed fuzzy partitions $\Pi_{k}^{p}$ corresponding to the properties of fineness (a), contrast (b) and directionality $(\mathrm{c})$.

Figure 2 shows the fuzzy partition $\Pi_{k}^{p}$ for the measure of Correlation [14] in the case of coarseness, and the measures of Tamura [10] in the case of contrast and directionality, which, according to our study, are the ones with the highest capacity to discriminate between different classes of the corresponding property (five in each case).

\section{Fuzzy Descriptor}

To the best our knowledge reaches, in the literature there is no formal definition of fuzzy descriptor, but ad-hoc measures intuitively based on fuzzy definitions extension of the concept of classic descriptor. In this section we propose a formal definition of descriptor, and specific measures for comparing descriptors.

We define a fuzzy descriptor as a level-two fuzzy set as follows:

Definition 3.1 Let $P$ be a universe of finite reference fuzzy sets representing imprecise visual properties or characteristics attributable to a pixel or region of an image and $v$ a visual concept associated to $P$. A fuzzy descriptor of $P$ based on a visual concept $v$ is defined as

$$
F D=\sum_{\widetilde{p} \in P} v(\widetilde{p}) / \widetilde{p}
$$

where $v(\widetilde{p}) \in[0,1]$ is the degree of fulfilment of the visual concept $v$ on $\widetilde{p}$.

Note that a fuzzy descriptor can be obtained from an image by calculating the degree to which each $\widetilde{p}$ over the pixels and/or regions of the image, and measuring the degree of fulfillment of $v$ to $\widetilde{p}$ in the whole image given by the fuzzy modelling of each visual feature. In this paper, we will consider four types of visual features: color, and the texture properties coarseness, directionality and contrast.

\subsection{Comparison measures between fuzzy descriptors}

Comparison measures between descriptors are necessary to perform any operation with them. In the next sections, fuzzy inclusion and similarity measures between two descriptors (sections 3.1.1 and 3.1 .2 , respectively) and fuzzy inclusion and similarity measures between sets of descriptors (section 3.1.3) are proposed.

\subsubsection{Inclusion between two fuzzy descriptors}

This section provides a way to calculate the degree of inclusion of a fuzzy descriptor $F D^{i}$ in $F D^{j}$ is proposed. The calculus is done using the Resemblance Driven Inclusion Degree introduced in [15], which computes the inclusion degree of two fuzzy sets whose elements are imprecise.

Definition 3.2 Let $F D^{i}$ and $F D^{j}$ be two fuzzy descriptors defined over a finite reference universe of fuzzy sets $P$, let $S$ be a similarity relation defined over the elements of $P, \otimes$ a t-norm, and $J$ a fuzzy implication operator. The inclusion degree of $F D^{i}$ in $F D^{j}$ driven by the similarity relation $S$, the $t$ norm $\otimes$ and the fuzzy implication operator $J$ is calculated as follows:

$$
\Theta_{S, \otimes, J}\left(F D^{j}, F D^{i}\right)=\underset{\widetilde{x} \in P}{\min } \max _{\widetilde{y} \in P} \theta_{S, \otimes, J}^{i, j}(\widetilde{x}, \widetilde{y})
$$

where

$$
\theta_{S, \otimes, J}^{i, j}(\widetilde{x}, \widetilde{y})=\otimes\left(J\left(F D^{i}(\widetilde{x}), F D^{j}(\widetilde{y})\right), S(\widetilde{x}, \widetilde{y})\right)
$$


In this paper, the minimum as t-norm $\otimes$, the similarity relation $S$ defined in equation 6 and the Lukasiewicz operator [16] as implication operator $J$ are used since it verifies all the axioms SinhaDougherty [17].

$$
S(\widetilde{x}, \widetilde{y})= \begin{cases}1 & \text { if } \widetilde{x}=\widetilde{y} \\ 0 & \text { otherwise }\end{cases}
$$

\subsubsection{Similarity between two fuzzy descriptors}

In this section the similarity degree between two Fuzzy Descriptors, $F D^{i}$ and $F D^{j}$ is defined. This similarity degree is calculated by means of the Generalized Resemblance between Fuzzy Sets proposed in [15], which is based on the concept of double inclusion.

Definition 3.3 Let $F D^{i}$ and $F D^{j}$ be two fuzzy descriptors defined over a finite reference universe of fuzzy sets $P$, let $S$ be a similarity relation defined over the elements of $P, \otimes_{1}$ and $\otimes_{2}$ two t-norms, and $J$ a fuzzy implication operator. The similarity degree of $F D^{i}$ in $F D^{j}$ is calculated as follows:

$$
\begin{aligned}
& \Phi_{S, \otimes_{1}, \otimes_{2}, J}\left(F D^{i}, F D^{j}\right)= \\
& \otimes_{1}\left(\Theta_{S, \otimes_{2}, J}\left(F D^{j}, F D^{i}\right), \Theta_{S, \otimes_{2}, J}\left(F D^{i}, F D^{j}\right)\right)
\end{aligned}
$$

We will use again as the minimum t-norm, both in $\otimes_{1}$ and $\otimes_{2}$, as well as the similarity relation and inclusion operator mentioned in the previous section.

\subsubsection{Weighted inclusion and similarity between sets of fuzzy descriptors}

Let $F D S^{i}=\left\{F D_{1}^{i}, F D_{2}^{i}, \ldots, F D_{n}^{i}\right\}$ and $F D S^{j}=$ $\left\{F D_{1}^{j}, F D_{2}^{j}, \ldots, F D_{n}^{j}\right\}$ two sets of Fuzzy Descriptors, with $n \geq 2$ being the number of Fuzzy Descriptors in each set.

The weighted inclusion degree of $F D S^{i}$ in $F D S^{j}$ driven by the resemblance relation $S$ is calculated as follows:

$$
\begin{aligned}
& \widehat{\Theta}_{S, \otimes, J}\left(F D S^{j}, F D S^{i}\right)= \\
& \sum_{k=1}^{n} w_{k} \times \Theta_{S, \otimes, J}\left(F D_{k}^{j}, F D_{k}^{i}\right)
\end{aligned}
$$

with $w_{k}$ being a weight verifying $\sum_{k=1}^{n} w_{k}=1$.

The weighted similarity degree between $F D S^{i}$ and $F D S^{j}$ is calculated by means of the following formulation:

$$
\begin{aligned}
& \widehat{\Phi}_{S, \otimes_{1}, \otimes_{2}, J}\left(F D S^{i}, F D S^{j}\right)= \\
& \sum_{k=1}^{n} w_{k} \times \Phi_{S, \otimes_{1}, \otimes_{2}, J}\left(F D_{k}^{i}, F D_{k}^{j}\right)
\end{aligned}
$$

with $w_{k}$ being a weight verifying $\sum_{k=1}^{n} w_{k}=1$.

It can be noticed that the values of the weights $w_{k}, k=1, \ldots, n$ can be adjusted to give more or less importance to the different Fuzzy Descriptor.
Again we propose to use the t-norms, similarity relation and inclusion operator mentioned in the previous section.

\section{Dominance-based Fuzzy Descriptors}

There are large amount of visual concepts based on visual properties, such as color or texture, that can be used to describe an image. One of the visual concepts used in the literature is the dominance of a visual property in an image. For example, color dominance or directionality dominance, which are directly related to the frequency of occurrence of such property in the image, although other aspects are considered.

Dominance is an imprecise concept, i.e., in an image it is possible in general to find colors/textures that are clearly dominant, colors/textures that are clearly not dominant, and colors/textures that are dominant to a certain degree, that depends on the percentage of pixels where the color/texture appears.

In this paper the dominant fuzzy concept is defined by a nondecreasing fuzzy quantifier, which is a natural way of representing the semantics of the concept dominant based on the number of pixels of a certain visual property. As we discuss in previous work [18], cardinality based on gradual numbers and a quantifier of type "approximately $x \%$ or more" and techniques to evaluate quantified sentences proposed provide a convenient way to obtain a degree of dominance.

Definition 4.1 Let $Q_{x}^{+}$be a nondecreasing fuzzy quantifier "approximately $x \%$ or more" and $\widetilde{p}$ a visual property. The dominance of $\widetilde{p}$ based on $Q_{x}^{+}$, $\operatorname{Dom}(\widetilde{p}) \in[0,1]$ in the image $I$ is defined as the result of evaluating the quantified sentence " $Q_{x}^{+}$of $I$ are $I_{\widetilde{p}}$ ", where $I_{\widetilde{p}}$ is the fuzzy sets of pixels of $I$ compatible with $\widetilde{p}$.

Considering the notion of "dominant" given in the definition 4.1 and the Fuzzy Descriptor defined in section 3, we propose two fuzzy descriptors that model the concepts of dominance on color and texture properties in an image.

In the case of color property, we define the following fuzzy descriptor for dominant colors:

Definition 4.2 Let $P=\widetilde{\Gamma}$ a fuzzy color space. A Fuzzy Dominant Color Descriptor is a level-two fuzzy set,

$$
F D_{C D}=\sum_{\widetilde{C} \in \widetilde{\Gamma}} \operatorname{Dom}(\widetilde{C}) / \widetilde{C}
$$

where $\operatorname{Dom}(\widetilde{C}) \in[0,1]$ is a degree of dominance associate to $\widetilde{C}$ in an image given by definition 4.1.

In the case of the texture property, we define the following fuzzy descriptor: 


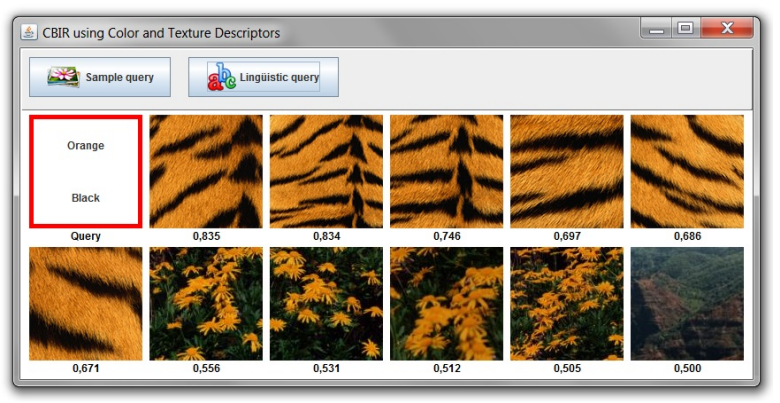

(a)

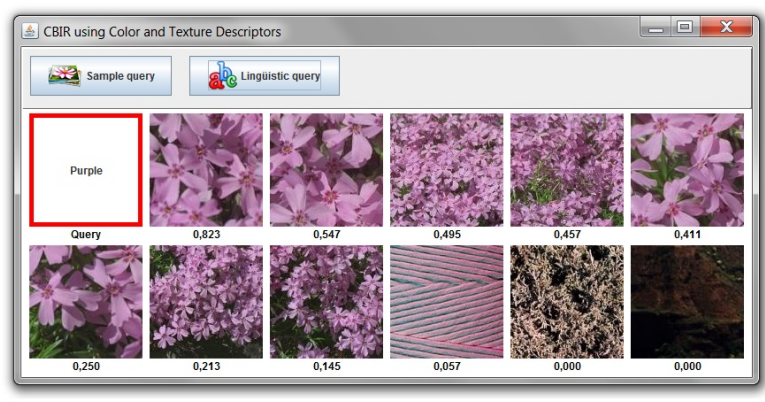

(b)

Figure 3: Retrieval results for the inclusion query using only color information with the labels (a) Orange and Black, and (b) Purple.

Definition 4.3 Let $P=\mathscr{T}$ a finite reference universe of texture fuzzy sets which model coarseness, directionality and contrast properties. We define the Fuzzy Dominant Texture Descriptor as the fuzzy set

$$
F D_{T D}=\sum_{T \in \mathscr{T}} \operatorname{Dom}(T) / T
$$

with $\operatorname{Dom}(T)$ being the dominance degree of a texture property $T$ given by definition 4.1.

For example, a Fuzzy Dominant Color Descriptor $F D_{C D}=1.0 /$ blue $+0.75 /$ red $+0.5 /$ yellow, it could be interpreted as fuzzy color blue dominates with degree 1 , red with degree 0.75 and yellow with degree 0.5. And, in the case of a Fuzzy Dominant Texture Descriptor $F D_{T D}=1.0 /$ fine $+0.8 /$ VeryDirectional $+0.2 /$ MediumContrast, could be interpreted as the texture properties fine dominates with degree 1, VeryDirectional with degree 0.8 and MediumContrast with degree 0.2.

\section{Retrieval Examples}

In this section, we will use the proposed dominancebased fuzzy descriptors $F D_{C D}$ and $F D_{T D}$ and the fuzzy inclusion and resemblance measures in a retrieval system. The database used in this retrieval system will be VisTex [19]. This database has been chosen because it is composed by a great variety of color texture images. Moreover, the standard VisTex database has been extended to incorporate images with more variety of colors and presence de-

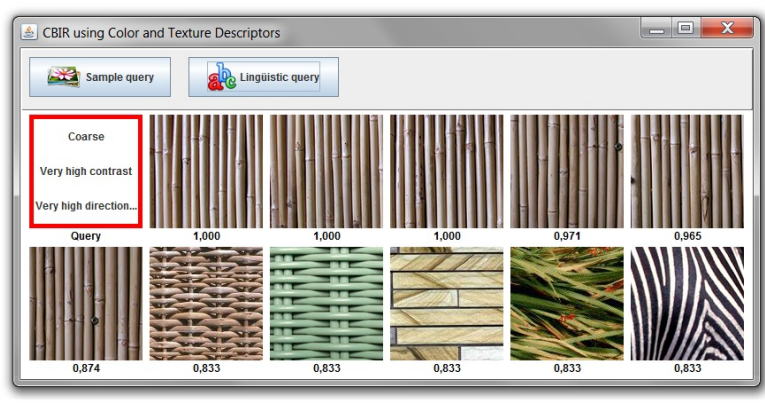

(a)



(b)

Figure 4: Retrieval results for the inclusion query using only texture information with the labels (a) Coarse, Very high contrast and Very high directionality, and (b) Very fine, Very high contrast and Very low directionality.

grees of the perceptual texture properties. In particular, the standard database is composed by 669 images of size $128 \times 128$, and it has been extended with 137 images of the same size.

Four retrieval examples will be shown in order to illustrate the performance of the system. In the first two examples, linguistic labels corresponding to color information and texture information are used separately as query, while in the next two examples, a combination of color and texture linguistic labels are employed. In our experiments we have chosen and empirically fixed the quantifier $Q_{x}^{+}$proposed in definition 4.1 with the values $x=0.25$ in the case of color, and $x=0.4$ in the case of texture according to our perception.

Figure 3 shows two retrieval results using only color information. In the first example (Figure $3(\mathrm{a})$ ), the linguistic labels Orange and Black have been used in the color inclusion query (specifically, the query descriptor is $F D_{C D}^{q u e r y}=1 /$ Orange + $1 /$ Black). Retrieved images are shown in decreasing order of the similarity degree to the query, that is shown below each image. It can be noticed that the six images of "tiger skin" included in the database, where orange and black colors are very dominant, appear in the top positions of results. The similarity degree of the next retrieved images (the "orange flowers") is lower, as the dominance of these colors decreases. Another retrieval result using color information can be shown in Figure 3(b). In this 


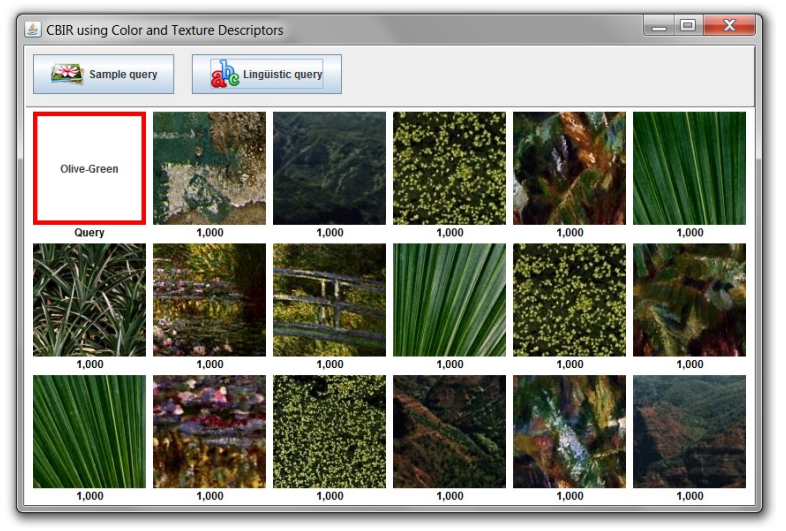

(a)

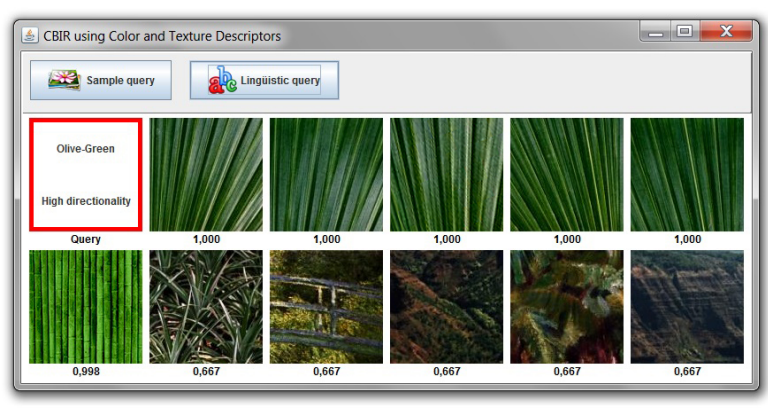

(b)

Figure 5: Retrieval results for (a) the color similarity query with the label Olive-Green, and (b) the color and texture similarity query with the labels Olive-Green and High directionality.

case, the query descriptor $F D_{C D}^{q u e r y}=1 /$ Purple has been used in order to retrieve the images of "purple flowers".

Figure 4 shows two retrieval results using only texture information. In the first example (Figure 4(a)), the linguistic labels Coarse, Very high contrast and Very high directionality have been used in the texture inclusion query (specifically, the query descriptor is $F D_{T D}^{q u e r y}=1 /$ Coarse $+1 /$ veryHighContrast + $1 /$ veryHighDirectionality). As in the previous examples, retrieved images are also shown in decreasing order of the similarity degree to the query. It can be noticed that all the images of "bamboo canes", whose dominant texture is coarse, very contrasted and very directional, can be found in the top positions of results. Another retrieval result using texture information is shown in Figure 4(b). In this case, the query descriptor $F D_{T D}^{\text {query }}=1 /$ veryFine + 1 /veryHighContrast +1 /veryLowDirectionality has been used in order to retrieve the images of "dry grass".

In the previous experiments, color descriptors and texture descriptors have been used separately. However, there are retrieval examples where the combination of color and texture information is needed. Figures 5 and 6 show two retrieval examples where the set of fuzzy descriptors $F D S=$

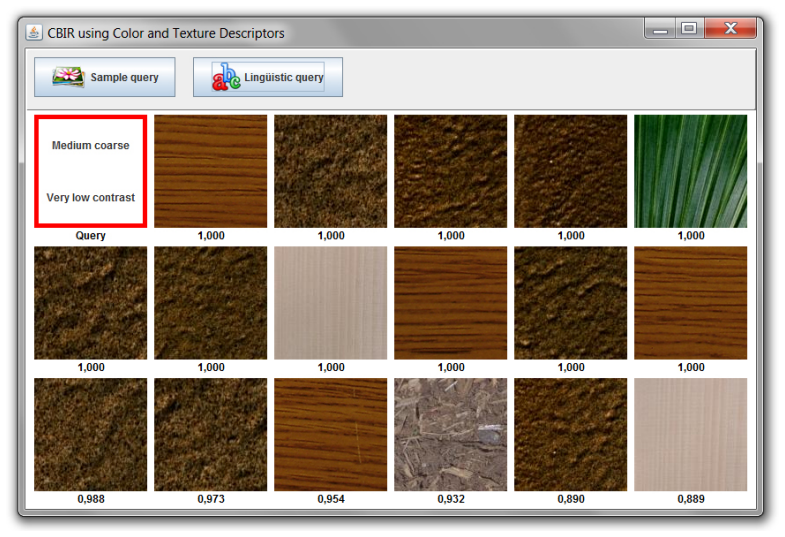

(a)

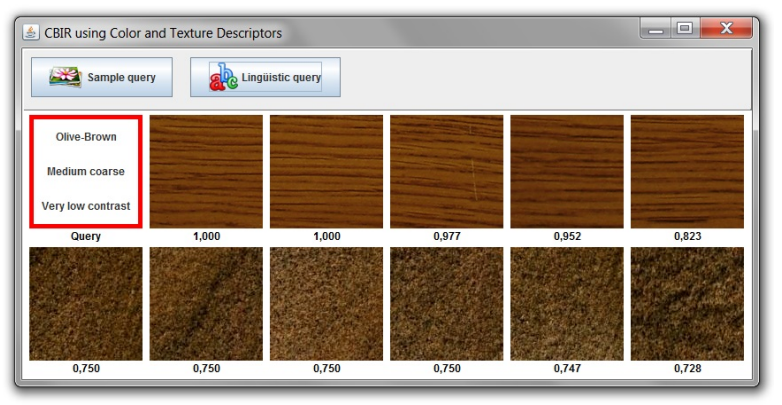

(b)

Figure 6: Retrieval results for (a) the texture similarity query with the labels Medium coarse and Very low contrast, and (b) the color and texture similarity query with the labels Olive-Brown, Medium coarse and Very low contrast.

$\left\{F D_{C D}, F D_{T D}\right\}$ is employed. In the first one, Figure 5 (a) shows the retrieval results using only color information. The linguistic label Olive-Green has been used in this color inclusion query, and it can be noticed that images with the same dominant colors can be found in these results, including "palm leaves", "landscapes", "paintings", etc.. This query can be refined by adding texture information. In the retrieval results shown in Figure 5(b), the linguistic labels Olive-Green and High directionality are used in the query for color and texture information, respectively (i.e., $F D_{C D}^{q u e r y}=1 /$ Olive - Green and $F D_{T D}^{q u e r y}=1 /$ highDirectionality). The similarity degree is calculated by using the fuzzy similarity measure shown in section 3.1.3 with $w_{1}=w_{2}=0.5$, i.e. giving equal importance to color and texture descriptors. It can be shown that in this case the "palm leaves" images are retrieved with the greatest resemblance degrees, because their textures are perceived as high directional.

In the last experiment, Figure 6(a) shows the retrieval results using only texture information. The linguistic labels Medium coarse and Very low contrast have been used as query, retrieving images with this type of texture. More specific retrieval results can be obtained by combining color and texture information, as it is shown in Figure 6(b), 
where the linguistic labels Medium coarse, Very low contrast and Olive-Brown have been used as query $\left(F D_{T D}^{q u e r y}=1 /\right.$ mediumCoarse $+1 /$ veryLowContrast and $F D_{C D}^{q u e r y}=1 /$ Olive - Brown). It can be noticed that in this case the "wood" images can be found in the top positions of results.

\section{Conclusions}

In this paper, the concept of Fuzzy Descriptor as a level-two fuzzy set over fuzzy features in an image has been presented. Particular propositions of fuzzy descriptor based on the dominance of color (Fuzzy Dominant Color Descriptor) and texture (Fuzzy Dominant Texture Descriptor) have been proposed. The dominance of each fuzzy feature (color and texture) has been calculated on the basis of a fuzzy quantifier representing the notion of dominance, and a fuzzy histogram based on gradual numbers representing as a fuzzy quantity the percentage of pixels that match each fuzzy feature. Fuzzy comparison measures over the new descriptors have been defined to allow inclusion-based and similarity-based conditions in image retrieval queries. Thus, the proposed framework has made our database system able to answer queries using color-based and texture-based linguistic labels in natural language. The system has been applied to texture image retrieval in order to analyze its performance, obtaining satisfactory results.

\section{ACKNOWLEDGMENT}

This work has been partially supported by the Spanish Government under the project TIN201458227 "DESCRIPCION LINGUISTICA DE INFORMACION VISUAL MEDIANTE TECNICAS DE MINERIA DE DATOS Y COMPUTACION FLEXIBLE".

\section{References}

[1] R.R. Korfhage. Information Storage and Retrieval. Wiley and Sons, 1997.

[2] K.P. Chung, J.B. Li, C.C. Fung, and K.W. Wong. A parallel architecture for feature extraction in content-based image retrieval system. IEEE Conf. on Cybernetics and Intelligent Systems, 1:468-473, 2004.

[3] C.C. Hsu, W.W. Chu, and R.K. Taira. A knowledge-based approach for retrieving images by content. IEEE Transactions on Knowledge and Data Engineering, 8(4):522532, 1996.

[4] D. Sánchez, J. Chamorro-Martínez, and M.A. Vila. Modelling subjectivity in visual perception of orientation for image retrieval. Information Processing and Management, 39:251-266, 2003.

[5] A. Younes, I. Truck, and H. Akdag. Color image profiling using fuzzy sets. Turkish Journal of Electrical Engineering and Computer Sciences, 13(3):343-359, 2005.

[6] J. Chamorro-Martínez, J.M. Medina, C. Barranco, E. Galán-Perales, and J.M. SotoHidalgo. Retrieving images in fuzzy objectrelational databases using dominant color descriptors. Fuzzy Sets and Systems, 158(3):312324, February 2007.

[7] A. Younes, I. Truck, and H. Akdag. Image retrieval using fuzzy representation of colors. Soft Comput., 11(3):287-298, 2006.

[8] J.M. Soto-Hidalgo, J. Chamorro-Martínez, and D. Sánchez. A new approach for defining a fuzzy color space. In IEEE World Congress on Computational Intelligence (WCCI 2010), pages 292-297, July 2010.

[9] K.L. Kelly and D.B. Judd. The iscc-nbs method of designating colors and a dictionary of color names. National Bureau of Standards (USA), (NBS Circular 553), 1955.

[10] H. Tamura, S. Mori, and T. Yamawaki. Textural features corresponding to visual perception. IEEE Trans. on Systems, Man and Cybernetics, 8:460-473, 1978.

[11] H. C. Lin, C. Y. Chiu, and S. N. Yang. Finding textures by textual descriptions, visual examples, and relevance feedbacks. Pattern Recognition Letters, 24(14):2255-2267, 2003.

[12] W. T. Lin, C. H. Lin, T. H. Wu, and Y. K. Chan. Image segmentation using the k-means algorithm for texture features. In Proceedings of the International Conference on Computer, Electrical, and Systems Science, and Engineering (ICCESSE), pages 26-28, 2010.

[13] J. Chamorro-Martínez, P. Martínez-Jiménez, and J. M. Soto-Hidalgo. On fuzzy partitions for visual texture modelling. In Proceedings of the 2011 IEEE International Conference on Fuzzy Systems, pages 759-765, Taipei, Taiwan, 2011.

[14] R. M. Haralick. Statistical and structural approaches to texture. Proceedings IEEE, 67(5):786-804, 1979.

[15] N. Marín, J.M. Medina, O. Pons, D. Sánchez, and M.A. Vila. Complex object comparison in a fuzzy context. Information and Software Technology, 45(7):431-444, 2003.

[16] C. Cornelis, C. Van der Donck, and E.E. Kerre. Sinha-Dougherty approach to the fuzzification of set inclusion revisited. Fuzzy Sets and Systems, 134:283-295, 2003.

[17] D. Sinha and E.R. Dougherty. Fuzzification of set inclusion: Theory and applications. Fuzzy Sets and Systems, 55(1):15 - 42, 1993.

[18] J. Chamorro-Martínez, D. Sánchez, J.M. SotoHidalgo, and P.M. Martínez-Jiménez. A discussion on fuzzy cardinality and quantification. some applications in image processing. Fuzzy Sets and Systems, 257(0):85 - 101, 2014.

[19] Technology Massachusetts Institute. Vision texture database. 